\title{
A local adaptive method for the numerical approximation in seismic wave modelling
}

\author{
Bruno G. Galuzzi ${ }^{1 *}$, Elena Zampieri ${ }^{2}$, Eusebio M. Stucchi ${ }^{1}$ \\ ${ }^{1}$ Department of Earth Sciences, University of Milan, Milan, Italy \\ ${ }^{2}$ Department of Mathematics, University of Milan, Milan, Italy \\ *Email address for correspondence: bruno.galuzzi@unimi.it \\ Communicated by Giorgio Fotia \\ Received on 12 20, 2016. Accepted on 10 09, 2017.
}

\begin{abstract}
We propose a new numerical approach for the solution of the $2 \mathrm{D}$ acoustic wave equation to model the predicted data in the field of active-source seismic inverse problems. This method consists in using an explicit finite difference technique with an adaptive order of approximation of the spatial derivatives that takes into account the local velocity at the grid nodes. Testing our method to simulate the recorded seismograms in a marine seismic acquisition, we found that the low computational time and the low approximation error of the proposed approach make it suitable in the context of seismic inversion problems.

\footnotetext{
Keywords: $\quad$ Finite difference; Seismic modelling; Seismic inversion;
} Optimization; Acoustic wave equation

AMS subject classification: 65L12, 65L20, 65Z05, 86A15, 86A22, 81U30
\end{abstract}

\section{Introduction}

Nowadays, in seismic exploration, it is common to formulate the problem of estimation of a high-resolution subsurface model as an optimization problem in which the difference between the observed and the predicted seismograms is minimized applying optimization algorithms (e.g., [1,2]). The predicted data can be obtained by the numerical solution of the wave equation using suitable numerical schemes, with the aim of improving either the approximation error or reducing the computation cost.

If we use finite differences to discretize the space domain, the approximation error depends mainly on the space step size $d x$ and the order $p$ of approximation of spatial derivatives. The main relation that links these two parameters to the physical parameters of the model is the numerical dispersion inequality, which limits the choice of $d x$ and $p$ on the basis of the minimum velocity of the model. However, if we use the same order $p$ for all the grid points of the spatial domain, the efficiency decreases and 


\section{B. Galuzzi, E. Zampieri, E. Stucchi}

the computational time increases. As a matter of fact the numerical dispersion depends on the local velocity, but the constraint associated to the numerical dispersion is related to the minimum velocity in the whole spatial domain (see [3]). See also [4] for a review of the recent developments in finite-difference modelling of seismic wave propagation and earthquake motion.

In this paper we describe a new numerical approach for the solution of the acoustic wave equation that optimizes the numerical dispersion inequality taking into account the local velocity at the grid nodes. According to the proposed method we reduce the computational time without increasing the approximation error. We tested our method to find an efficient numerical solution, (i.e. a seismogram), in the case of a simulated seismic acquisition. The lower computational time required for a given value of the approximation error makes this approach suitable for a computationally expensive seismic modelling or inversion problem.

\section{A short summary of seismic exploration}

Seismic exploration, which is widely used in search of oil and gas reservoirs, aims at the estimation of elastic properties of subsurface using techniques based on active seismic data (e.g., [5,6]). In Figure 1 (left) we show a sketch of a 2D marine seismic acquisition, for studying the geological structures of a given area situated below the sea floor. An exploration ship pulls an energy source and a set of receivers close to the sea surface. The source consists of a device (air-gun) that generates controlled seismic energy, i.e., a band-limited time-varying impulse signal. The set of receivers consists of hydrophones, able to listen the pressure variation in the water, then recorded by a sismometer. Generally, the receivers are located at increasing uniform distance from the source, forming a listening spread moving over the study-area. When the source is activated, the seismic waves propagate firstly in the water and then in the rocks situated below the sea floor. The different elastic properties of the rocks cause different responses at the passage of seismic waves. By recording the reflection and refraction waves propagating towards the receivers, it is possible to obtain information concerning the properties of the area under investigation. In Figure 1 (right) we show some typical parameters of a seismic acquisition according to which the considered area can measure up to $10-20$ kilometers in depth and up to hundreds of kilometers in length. Therefore, it is not possible to acquire sufficient information from a single activation of the source, i.e., from a single seismic shot. This is due to different reasons, e.g., the limited energy of the source, the different kinds of recorded noise, the complexity of the 


\section{A local adaptive method for numerical approximation in seismic modelling}

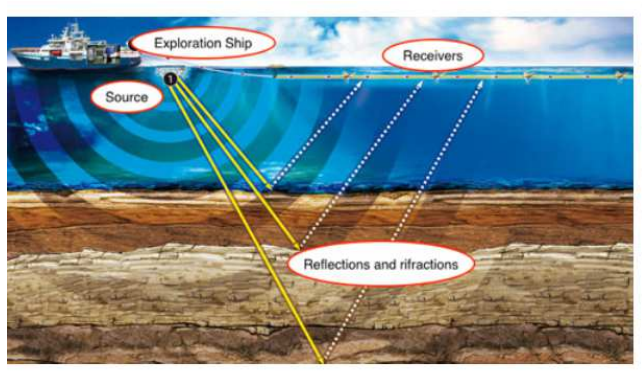

\begin{tabular}{|c|c|}
\hline \multicolumn{2}{|c|}{ Geological model } \\
\hline Parameters & Range \\
\hline Length & {$[1 \mathrm{Km}, 100 \mathrm{Km}]$} \\
\hline Depth & {$[0.1 \mathrm{Km}, 10 \mathrm{Km}]$} \\
\hline \multicolumn{2}{|c|}{ Acquisition parameters } \\
\hline Shots & {$[100,1000]$} \\
\hline Receivers & {$[100,1000]$} \\
\hline Registration time & {$[1 \mathrm{~s}, 10 \mathrm{~s}]$} \\
\hline Temporal frequencies & {$[1 \mathrm{~Hz}, 200 \mathrm{~Hz}]$} \\
\hline
\end{tabular}

Figure 1. (left) An example of a marine seismic acquisition; (right) parameters of a $2 D$ seismic acquisition. (Note that these parameters are indicative and they depend on the objectives of the survey).

subsurface geological model, the equipment's limitations. As a remedy to this inconvenience, a huge number of seismic shots is acquired moving the location of the source and the receivers along the investigated area. In a marine seismic acquisition, when the source is activated, each receiver starts recording any pressure variation. We call seismic trace the record of a single receiver (see Figure 2, a)). Generally, an exploration seismogram is formed by the gather of all the seismic traces (see Figure 2, b)) of a single shot, ordered as a function of the distance from the source. This distance is known in the specialized literature as offset. Thus, a seismogram is a data matrix in which each row represents the values recorded by all the receivers at a particular time. We define sampling rate the constant time interval elapsed between the acquisition of successive rows. In this context, the aim of the seismic exploration is to estimate a model of lateral and vertical variations of the elastic properties of subsurface, such as the $\mathrm{P}$-wave velocity $v_{p}(\vec{x})$, the S-wave velocity $v_{s}(\vec{x})$ and the density $\rho(\vec{x})$. The more the properties of the rocks in the study-area are different, the more complex the seismograms are.

This is the reason why a direct interpretation of the seismograms is not possible and it is necessary to formulate this problem as an inverse problem. Calling $d_{o b s}$ the set of the acquired seismograms and $d_{\text {pred }}\left(m_{\text {pred }}\right)$ the set of predicted seimograms, obtained by the numerical solution of the wave equation as a function of the predicted model $m_{\text {pred }}$, the idea is to find the model $\bar{m}$ such that:

$$
d_{\text {pred }}(\bar{m})=d_{\text {obs }}
$$

A perfect match between the predicted and the observed data is not possible for several reasons, such as the presence of the noise in the observed data, 


\section{B. Galuzzi, E. Zampieri, E. Stucchi}
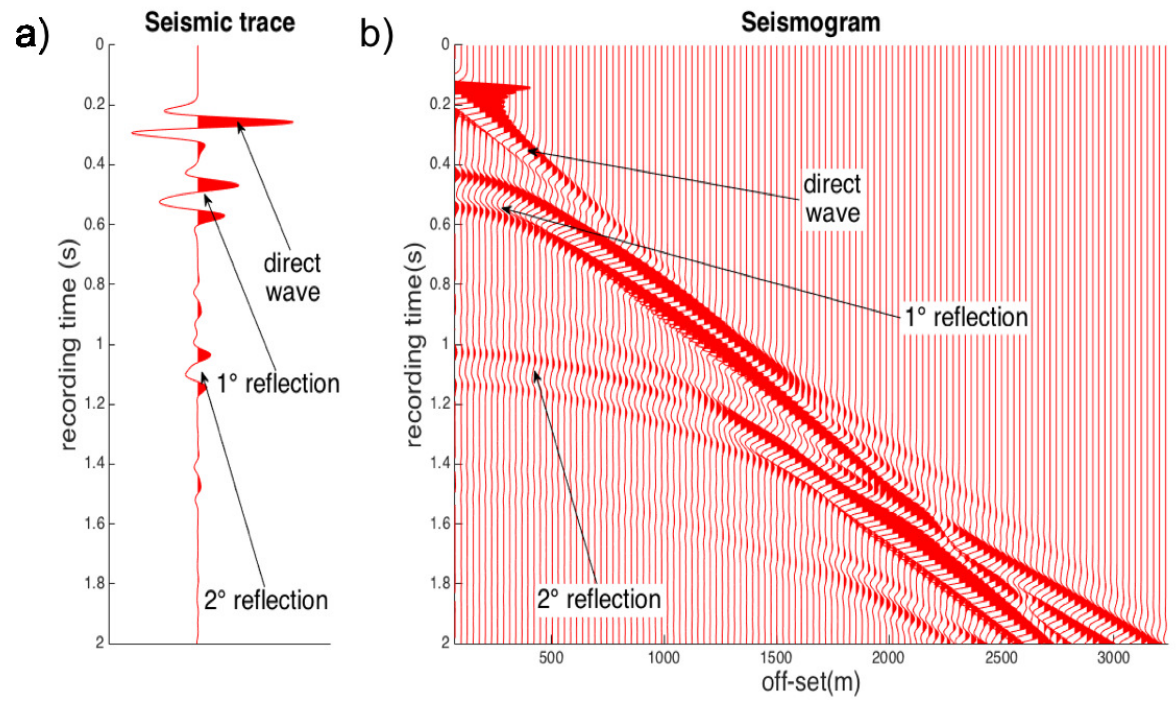

Figure 2. a) A seismic trace; b) a seismogram given by gathering all the seismic traces of a single shot.

the approximation error of the numerical solution, and the constraints of the approximating mathematical model used to describe the generation and propagation of seismic waves.

Therefore, this problem can be formulated as a minimization problem, that reads as follows: if we consider a misfit function $f\left(m_{\text {pred }}\right)$ measuring the differences between the predicted and the observed data

$$
f\left(m_{\text {pred }}\right)=\left\|d_{\text {pred }}\left(m_{\text {pred }}\right)-d_{\text {obs }}\right\|,
$$

where $\|\cdot\|$ is a suitable norm, we want to determine a model $\bar{m}$ that minimizes the misfit function

$$
\bar{m}=\underset{m_{\text {pred }} \in M}{\arg \min } f\left(m_{\text {pred }}\right),
$$

where $M$ is the set of all possible predicted models. The number of unknowns of the misfit function depends on the spatial discretization used to approximate the predicted model $m_{\text {pred }}$ and, in general, hundreds or thousands of unknowns can be necessary if the study-area is large, even in a $2 \mathrm{D}$ case.

Since the misfit function is generally a non-linear function of the predicted model, an iterative minimization procedure is necessary to obtain a good 


\section{A local adaptive method for numerical approximation in seismic modelling}

approximation of the global minimum. This problem is known as Full Waveform Inversion (FWI) (see, e.g., [2,7]). Many local or global different optimization strategies have been studied in recent years. However, such procedures can require a huge number of forward modellings to obtain acceptable results $[8,9]$. This is the reason why an efficient numerical solution of the wave equation is crucial in order to compute the predicted data. In particular, the solution should provide a low approximation error for the seismic data using a low computational time.

\section{The $2 \mathrm{D}$ acoustic seismic equation and its numerical model}

The mathematical model of generation and propagation of seismic waves within the Earth depends on the particular seismic problem we set up. In particular, in this work we want to model the seismograms of an activesource marine seismic acquisition. Here we assume only P-wave propagation and heterogeneous density data that are small if compared to pressure and source term variations. In this case [3,7], the seismic wave propagation can be approximated by the following equation:

$$
\ddot{p}(\vec{x}, t)=v_{p}(\vec{x})^{2} \Delta p(\vec{x}, t)+f(\vec{x}, t),
$$

where $t \in[0, T]$ is the recording time, $\vec{x} \in D(\vec{x}) \subseteq \mathbb{R}^{3}$ is the space domain of propagation of seismic wave, $p$ is the acoustic pressure of the wave, $f$ is the seismic source and $v_{p}$ is the acoustic wave velocity. A realistic range for wave speed can be between $1500 \mathrm{~m} / \mathrm{s}$ (water) and $6000 \mathrm{~m} / \mathrm{s}$ (granite). The support of the seismic source $f$ is much smaller than that of the geological medium. Moreover, in this work we consider $f$ to be due to an isotropic $P$ wave source. Consequently, it can be approximated by a $\delta$-source in space

$$
f(\vec{x}, t)=\delta\left(\vec{x}-\vec{x}_{0}\right) s(t),
$$

where $s$ is the seismic wavelet, describing the variation of the seismic source in time. We set $t=0$ the time value when the source is activated, with $p(\vec{x}, 0)=\dot{p}(\vec{x}, 0)=0, \forall \vec{x} \in D(\vec{x})$ as initial conditions. Since many sourcereceiver geometries are often confined to a plane (for example $y=0$ ), and because of the large computational cost of $3 \mathrm{D}$ modelling, in this work we consider only the $2 \mathrm{D}$ acoustic wave equation

$$
\ddot{p}(\vec{x}, t)=v_{p}(\vec{x})^{2} \Delta p(\vec{x}, t)+\delta\left(\vec{x}-\vec{x}_{0}\right) s(t),
$$

with $\vec{x} \in D(\vec{x}) \subseteq \mathbb{R}^{2}$. In general, a 2D modelling of wave propagation cannot be used to provide a direct quantitative comparison, including amplitude 


\section{B. Galuzzi, E. Zampieri, E. Stucchi}

information, with seismic data acquired along a line and when the model is assumed to be 2.5-D. However, there are many strategies that make the passage from $2 \mathrm{D}$ to $2.5-\mathrm{D}$ possible (e.g., [10-12]).

The acoustic wave equation can be written as a first order system of two equations

$$
\left\{\begin{array}{l}
\dot{v}(\vec{x}, t)=v_{p}(\vec{x})^{2} \Delta p(\vec{x}, t)+\delta\left(\vec{x}-\vec{x}_{0}\right) s(t) \\
\dot{p}(\vec{x}, t)=v(\vec{x}, t)
\end{array},\right.
$$

where

$$
\Delta p(\vec{x}, t) \equiv \frac{\partial^{2} p(\vec{x}, t)}{\partial x^{2}}+\frac{\partial^{2} p(\vec{x}, t)}{\partial z^{2}} .
$$

The semi-discrete numerical approximation is based on the Leap-Frog method (e.g., [13-16]), an explicit second order in time method with uniform time step $d t$, that reads:

$$
\left\{\begin{array}{l}
v(\vec{x})^{t+\frac{1}{2}}=v(\vec{x})^{t-\frac{1}{2}}+d t v_{p}(\vec{x})^{2} \Delta p(\vec{x})^{t}+d t \delta\left(\vec{x}-\vec{x}_{0}\right) s^{t} \\
p(\vec{x})^{t+1}=p(\vec{x})^{t}+d t v(\vec{x})^{t+\frac{1}{2}}
\end{array} .\right.
$$

The space domain $D$ is sampled with a uniform step $d x$ along the horizontal and vertical direction, obtaining a regular grid $\left\{D_{i, j}\right\}$ built on $n x \cdot n z$ grid nodes, with $i=1, \ldots, n x, j=1, \ldots, n z, \vec{x}_{i, j}=\left(x_{i}, z_{j}\right)$, obtaining the finite difference system:

$$
\left\{\begin{array}{l}
v\left(\vec{x}_{i, j}\right)^{t+\frac{1}{2}}=v\left(\vec{x}_{i, j}\right)^{t-\frac{1}{2}}+d t v_{p}\left(\vec{x}_{i, j}\right)^{2} \Delta p\left(\vec{x}_{i, j}\right)^{t}+d t \delta\left(\vec{x}_{i, j}-\vec{x}_{0}\right) s^{t} \\
p\left(\vec{x}_{i, j}\right)^{t+1}=p\left(\vec{x}_{i, j}\right)^{t}+d t v\left(\vec{x}_{i, j}\right)^{t+\frac{1}{2}}
\end{array} .\right.
$$

In order to approximate the spatial derivatives, we implement a 2nd-order space operator [17]

$$
\frac{\partial^{2} p\left(\vec{x}_{i, j}\right)^{t}}{\partial x^{2}} \approx \frac{\sum_{k=1}^{n} c_{k}\left(p\left(\vec{x}_{i+k, j}\right)^{t}+p\left(\vec{x}_{i-k, j}\right)^{t}\right)}{d x^{2}}-2 \frac{\left(\sum_{k=1}^{n} c_{k}\right) p\left(\vec{x}_{i, j}\right)^{t}}{d x^{2}}
$$

where the $c_{i}$ 's are suitable coefficients computed in order to guarantee the $2 n$-order of approximation for spatial derivatives [18]. In Table 1 we recall the values of the coefficients $c_{i}$ 's as a function of the order of approximation $p=2 n$, for $n=1, \ldots, 6$.

We use a similar formula for the approximation of the spatial derivatives along the depth, with the same order of approximation $p$ for both the directions. An efficient way to implement the FD system consists in reordering 


\section{A local adaptive method for numerical approximation in seismic modelling}

Table 1. Coefficients $c_{i}$ 's of FD high order schemes.

\begin{tabular}{|c|c|c|c|c|c|c|}
\hline$p$ & $c_{1}$ & $c_{2}$ & $c_{3}$ & $c_{4}$ & $c_{5}$ & $c_{6}$ \\
\hline 2 & 1 & 0 & 0 & 0 & 0 & 0 \\
4 & $\frac{4}{3}$ & $-\frac{1}{12}$ & 0 & 0 & 0 & 0 \\
6 & $\frac{3}{2}$ & $-\frac{3}{20}$ & $\frac{1}{90}$ & 0 & 0 & 0 \\
8 & $\frac{8}{5}$ & $-\frac{1}{5}$ & $\frac{8}{315}$ & $-\frac{1}{560}$ & 0 & 0 \\
10 & $\frac{5}{3}$ & $-\frac{5}{21}$ & $\frac{5}{126}$ & $-\frac{5}{28}$ & $\frac{1}{3150}$ & 0 \\
12 & $\frac{12}{7}$ & $-\frac{15}{56}$ & $\frac{10}{189}$ & $-\frac{1}{112}$ & $\frac{2}{1925}$ & $-\frac{1}{16632}$ \\
\hline
\end{tabular}

the space grid nodes $\vec{x}_{i, j}$ as a row vector $\vec{x}_{k}, k=1, \ldots, n x \cdot n z$, scrolling the grid matrix along the rows. In this way we obtain:

$$
\left\{\begin{array}{l}
v\left(\vec{x}_{k}\right)^{t+\frac{1}{2}}=v\left(\vec{x}_{k}\right)^{t-\frac{1}{2}}+d t v_{p}\left(\vec{x}_{k}\right)^{2} \hat{\Delta} p\left(\vec{x}_{k}\right)+d t \delta\left(\vec{x}_{k}-\vec{x}_{0}\right) s^{t} \\
p\left(\vec{x}_{k}\right)^{t+1}=p\left(\vec{x}_{k}\right)^{t}+d t v\left(\vec{x}_{k}\right)^{t+\frac{1}{2}}
\end{array},\right.
$$

where $\hat{\Delta}$ is the finite difference Laplacian approximation, that corresponds to the action of a matrix $K_{\hat{\Delta}}$ on $p\left(\overrightarrow{x_{k}}\right)$, where the elements of each row are the coefficients obtained for the $p$-order approximation of the spatial derivatives. The matrix $K_{\hat{\Delta}}$ is sparse, symmetric and banded, with the number of non zeros elements $N_{\hat{\Delta}}$ that is of the order of $(2 p+1) \cdot n x$. $n z$. As a further difficulty, the reduction of the computational domain to only a part of the true physical domain causes the introduction of artificial reflecting boundaries. To avoid these artificial reflections we use the socalled Gaussian taper method [19]. It relies on the introduction of a thin strip along the artificial boundary and multiplying the solution, at each time step, by a Gaussian taper factor $G$ that is a function of the position. The modified system reads:

$$
\left\{\begin{array}{l}
v\left(\vec{x}_{k}\right)^{t+\frac{1}{2}}=G\left(\vec{x}_{k}\right)\left(v\left(\vec{x}_{k}\right)^{t-\frac{1}{2}}+d t v_{p}\left(\vec{x}_{k}\right)^{2} \hat{\Delta} p\left(\vec{x}_{k}\right)^{t}+d t \delta\left(\vec{x}_{k}-\vec{x}_{0}\right) s^{t}\right) \\
p\left(\vec{x}_{k}\right)^{t+1}=G\left(\vec{x}_{k}\right)\left(p\left(\vec{x}_{k}\right)^{t}+d t v\left(\vec{x}_{k}\right)^{t+\frac{1}{2}}\right)
\end{array},\right.
$$

with $G\left(\vec{x}_{k}\right)=1$ at grid nodes outside the absorbing boundary layer, $G\left(\vec{x}_{k}\right) \in$ $[0.92,1)$ at nodes inside the absorbing boundary layer. At external borders of absorbing boundary layers, we consider homogeneous Dirichlet boundary conditions.

\section{An adaptive scheme to reduce the numerical dispersion}

There are two main relations that generally influence the approximation error. The first is the well known CFL numerical stability relation (see [20]) 


\section{B. Galuzzi, E. Zampieri, E. Stucchi}

$$
d t<\frac{d x}{v_{\max }} \lambda
$$

where $\lambda=\lambda(p) \in[0.5,1]$ is the Courant number. This inequality bounds the maximum possible time step $d t$ as a function of the space step size $d x$ and of the maximum velocity $v_{\max }$. The second is the numerical dispersion relation [3]

$$
d x<\frac{v_{\min }}{n f_{\max }},
$$

where $n=n(p)$ is the number of grid points per wavelength. The grid dispersion limits the maximum possible space step as a function of the minimum velocity $v_{\min }$ and of the maximum frequency $f_{\max }$ of the source signal $s(t)$. Numerical stability is a necessary condition to implement any explicit finite difference method. Keeping into account the order of magnitude of the wave speed in rocks, the stability condition implies that the maximum possible time step must be numerically at least three orders of magnitude lower than the minimum possible space step size, when time and length are measured in seconds and meters (SI units), respectively. Consequently, the error of approximation is more sensitive to the spatial parameters $(d x, p)$ and to the numerical dispersion relation. In order to estimate the number of points for wavelength $n$ as a function of $p$ for a given numerical dispersion error, we consider the function $\cos \left(\frac{2 \pi x}{l}\right)$, where $l$ denotes the wavelength, and we calculate analytically the second derivative of this function at $x=0$. A numerical solution can be obtained by sampling the function with different sampling intervals $d x=\frac{l}{n}$ by using different $p$-order operators. Figure 3 , a) shows the percentage of the approximation error as a function of $n$ for different order $p$, while Table 2 lists the values of $n$ as a function of $p$ to obtain an error below $1 \%$. As we expect if we use a low order $p$, the number of points per wavelength increases, while if we use a high order $p$, it decreases until the theoretic limit of 2 (it is not possible to get $n<2$, because in this case the Nyquist-Shannon sampling theorem [21] would be violated).

Table 2. Values of $n$ as a function of $p$ such that the error is below $1 \%$.

\begin{tabular}{|c|c|c|c|c|c|c|c|c|c|c|c|c|}
\hline$p$ & 2 & 4 & 6 & 8 & 10 & 12 & 14 & 16 & 18 & 20 & 22 & 24 \\
\hline$n$ & 18 & 6.3 & 4.5 & 3.75 & 3.5 & 3.25 & 3 & 2.9 & 2.8 & 2.7 & 2.6 & 2.5 \\
\hline
\end{tabular}

Generally, in a seismic acquisition, the minimum velocity $v_{\min }$ and the maximum frequency $f_{\max }$ can be considered fixed parameters, estimated by 


\section{A local adaptive method for numerical approximation in seismic modelling}

a)

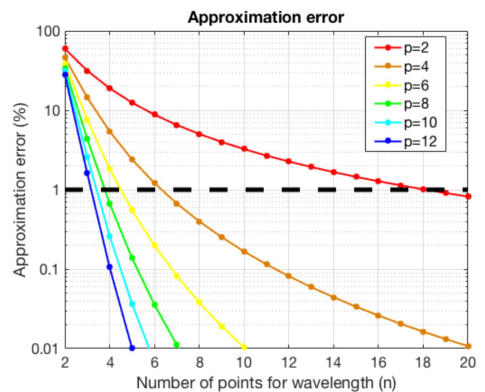

b)

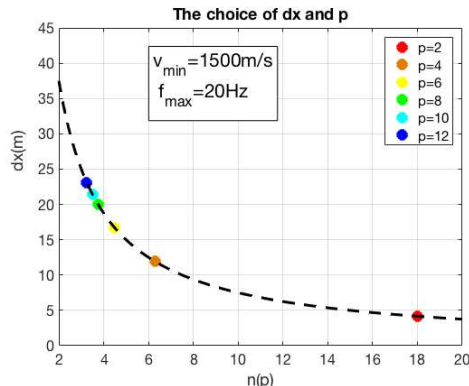

Figure 3. a) Approximation error as a function of the number of points for wavelength and the order of approximation of the partial derivatives $p$; b) choice of $p$ and $d x$ given the ratio $\frac{v_{\min }}{f_{\max }}$ to keep the approximation error below the desired value of $1 \%$.

the geological context and signal characteristics. For example, in a marine seismic acquisition survey, $v_{\min }=1500 \mathrm{~m} / \mathrm{s}$, that is the water velocity, and the maximum signal frequency $f_{\max }$ can be of the order of $150 \mathrm{~Hz}$. However, the maximum frequency used in the context of FWI to reduce the number of local minima in the objective function is of the order of $10-20 \mathrm{~Hz}$ (e.g., $[9,22])$. Considering the function

$$
y(p)=\frac{v_{\min }}{f_{\max } n(p)},
$$

with a fixed $\frac{v_{\min }}{f_{\max }}$ ratio, we can conclude that, in order to obtain a dispersion error lower than a given threshold, we must choose $p$ and $d x$ such that $d x<y(p)$. Such choices are represented by the colored dots on the black dashed line in Figure 3, b).

In particular, increasing the space sampling $d x$ causes an increase of the order $p$ and vice-versa. The choice of the right parameters $p$ and $d x$ is important not only to reduce the numerical dispersion error, but also for their influence on the computational time $t_{\text {comp }}$. Indeed, the computational time increases linearly with $p, n x$ and $n z$, since $t_{c o m p} \propto n x \cdot n z \cdot p$.

The numerical dispersion relation can cause very inefficient forward modelling for seismic inversion applications, when only the minimum velocity of the model is considered. An example of this fact can be observed in Figure 4 , a), where there is a velocity model formed by a constant velocity layer of $6000 \mathrm{~m} / \mathrm{s}$ superimposed by a thin layer where the velocity is $1500 \mathrm{~m} / \mathrm{s}$. The choice of the order $p$ and of the space sampling $d x$ is influenced only by the small part of the model having the lowest velocity value. The examined model is clearly unrealistic, but we have similar consequences also for realistic cases. For example, in a marine seismic acquisition generally the minimum velocity is the water velocity situated on the top of the studied 


\section{B. Galuzzi, E. Zampieri, E. Stucchi}
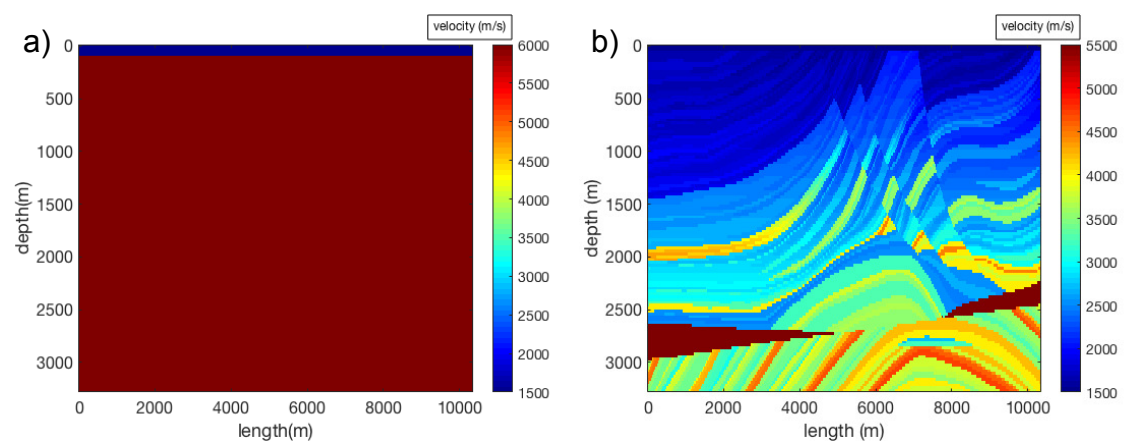

Figure 4. a) An example of a velocity model where the local low velocity can compromise the computational time; b) a synthetic but realistic complex geological model situated below a flat sea floor in which the mean velocity is higher than the water one.

region, and the geological structures situated below the sea floor have a higher acoustic velocity. In particular, Figure 4, b) shows the 2D acoustic Marmousi velocity model (see [23]), that is discussed more in details in Section 5. By these considerations the choice of a classical finite difference approach to model the seismic wave propagation is inefficient. To improve the method, we can follow two possible approaches. The first is to use a local velocity-dependent space sampling $d x_{l o c}$. By this method we could use a small space step size for those regions of the model with low velocity, and larger space sampling for those regions with higher velocity. Unfortunately, this method could be unsuitable for an inversion procedure such as the FWI, where the unknowns are the grid node velocities. The second approach consists in fixing a modelling grid, and using different local orders $p_{l o c}$ of approximation of spatial derivatives in different part of the model, instead of a global one $p$. Our implementation is based on this second approach and leads to an improved procedure.

To obtain a local order of approximation of the spatial derivatives, we start again with the numerical dispersion relation, setting $d x$ and $f_{\text {max }}$, and replacing $v_{\min }$ with $v_{p}$ (see Figure 5, a)):

$$
n<\frac{v_{\min }}{d x \cdot f_{\max }} \Rightarrow n_{l o c}<\frac{v_{p}}{d x \cdot f_{\max }} .
$$

This local dispersion inequality applied to the Marmousi velocity model of Figure 4, b), allows to compute the parameter $n_{l o c}$ (see Figure 5, a)) i.e., the local number of points for wavelength to be used for computing the solution. Then we can estimate the local order of approximation of the spatial derivatives $p_{l o c}$ (see Figure $5, \mathrm{~b}$ )) from $n_{l o c}$, given the desired approximation error. For instance $p_{l o c}$ can be chosen as the minimum order 


\section{A local adaptive method for numerical approximation in seismic modelling}

a)

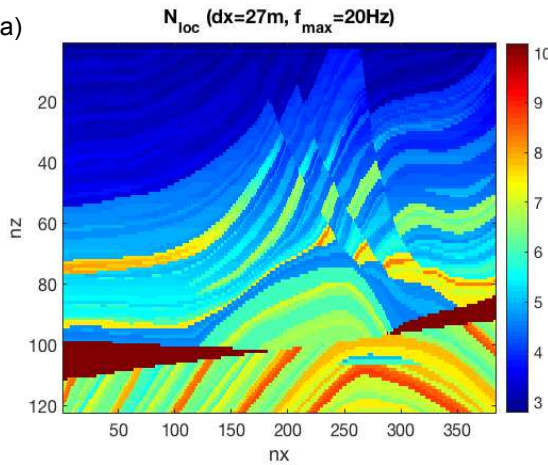

b)

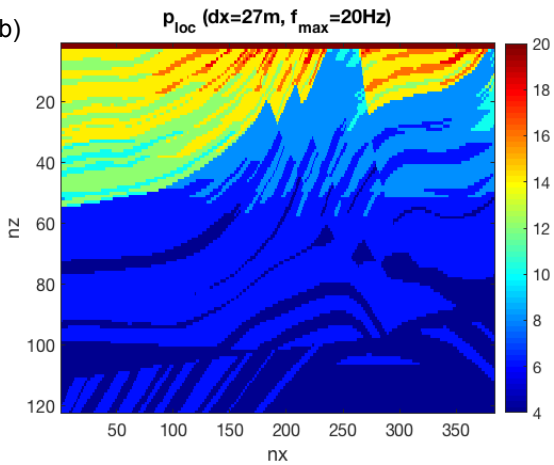

Figure 5. a) $n_{l o c}$ and b) $p_{l o c}$, for the Marmousi velocity model with $d x=27 \mathrm{~m}$ and $f_{\max }=20 \mathrm{~Hz}$.

that gives an error of approximation below $1 \%$ at the corresponding $n_{l o c}$ position (see Table 2). Therefore it will be high in those regions of the model with low velocities and low in those regions with high velocity values.
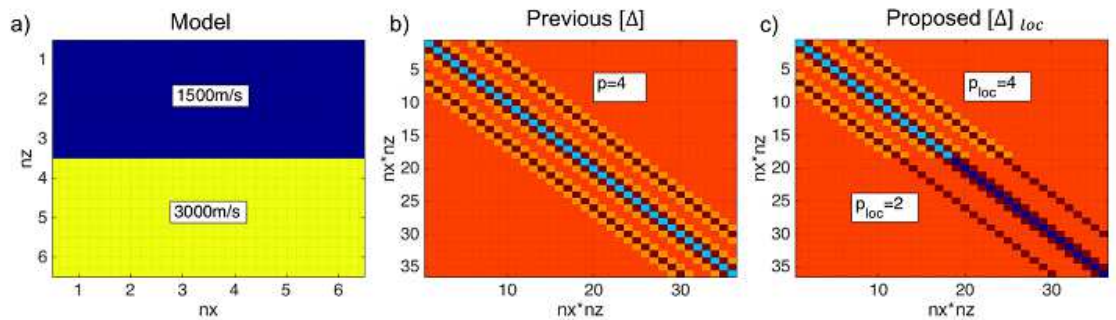

Figure 6. a) A simple model with two layers of different velocity; b) The Laplacian matrix using the classical numerical dispersion relation; c) the Laplacian matrix using the proposed method.

This new approach modifies the structure of the Laplacian approximation matrix $K_{\hat{\Delta}}$. Indeed, the new local Laplacian approximation $K_{\hat{\Delta}_{l o c}}$ depends on the local velocity, causing an asymmetric matrix. Besides, the number of non zero elements will depends on the model velocity and in general this quantity is smaller than in the classical approximation of the Laplacian operator, since it uses only the information regarding the minimum velocity. In particular we obtain:

$$
N_{\hat{\Delta}_{l o c}} \approx \sum_{i=1}^{\frac{p_{\max }}{2}}(2 \cdot 2 i+1) n_{2 i},
$$

where $p_{\max }$ is the maximum implemented $p$-order, $n_{2 i}$ is the number of grid nodes with $p_{l o c}=2 i$, and $n_{2}+n_{4}+\ldots+n_{p_{\max }}=n x \cdot n z$. In Figure 6, a) 


\section{B. Galuzzi, E. Zampieri, E. Stucchi}

we show an example of FD grid with $n x=n z=6$ and $d x=15 m, f_{\max }=$ $10 \mathrm{~Hz}$, and a model with two layers of different velocity: $1500 \mathrm{~m} / \mathrm{s}$ (top) and $3000 \mathrm{~m} / \mathrm{s}$ (bottom). In Figure $6 \mathrm{~b}$ ) and c) we show respectively the Laplacian approximation matrix based on the classical numerical dispersion relation and the same matrix for the method we propose. The classical approximation uses a global order $p=4$, while the proposed method uses $p=4$ for the first layer and $p=2$ for the second layer, where the velocity is higher. Note that the number of non-zero elements in the second matrix is smaller than in the previous one.

\section{Numerical test}

In this section we present an example to compute an efficient solution of the acoustic wave equation, that is a synthetic seismogram, using the approximation based on the classical numerical dispersion relation and our proposed method. Figure 7, a) shows the 2D acoustic Marmousi velocity
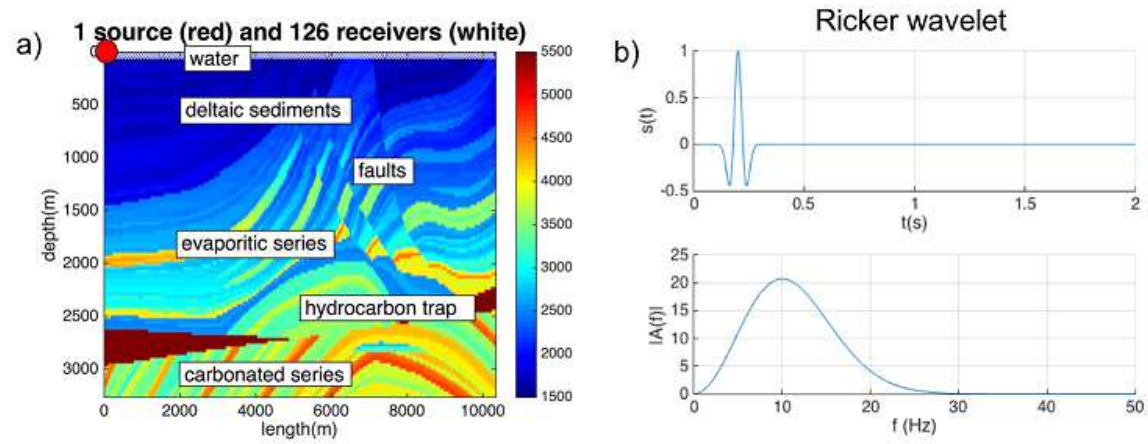

Figure 7. a) The Marmousi model, with the source-receiver layout used in the numerical test; b) the Ricker wavelet (top) and its spectrum (bottom).

model, typically used in the context of a seismic inversion and modelling procedure. It consists of 121 layers of rocks of various type, situated below a water layer of $54 \mathrm{~m}$. The acoustic velocity varies from a minimum of $1500 \mathrm{~m} / \mathrm{s}$ of the water layer to a maximum of $5500 \mathrm{~m} / \mathrm{s}$. The sea floor is flat, but many layers have high dip angles. Besides, in the center of the model, there is a series of normal faults. The main geological structures are indicated in Figure 7, a). The acquisition layout consists of a seismic source and 126 receivers, with a uniform spacing of $d x=81 \mathrm{~m}$. The depth of the source and of the receivers is $27 \mathrm{~m}$ below the sea surface (top of the model) and we set an offset of $162 \mathrm{~m}$ between the first receiver and the source. We use a Ricker wavelet as the source signal $s(t)$ (see Figure 7, b)), namely the negative normalized second derivative of a Gaussian function, with a peak 


\section{A local adaptive method for numerical approximation in seismic modelling}

frequency of $10 \mathrm{~Hz}$ and a frequency range between 0 and $30 \mathrm{~Hz}$. We set absorbing boundary conditions on lateral and bottom sides. On the contrary, on the top side we consider reflective boundary conditions to simulate the high velocity and density contrast between air and water.

We study the efficiency of the proposed method as a function of the numerical dispersion and the computational time. Figure 8 displays four different

Table 3. Numerical parameters and computational time for the four seismograms in Figure 8.

\begin{tabular}{|c|c|c|c|c|}
\hline$d t$ & $0.00025 s$ & $0.002 s$ & $0.002 s$ & $0.002 s$ \\
\hline$d x$ & $3 m$ & $27 m$ & $27 m$ & $27 m$ \\
\hline$p$ & 4 & 4 & $p_{l o c}$ & 24 \\
\hline time & $57 \mathrm{~m}$ & $6 \mathrm{~s}$ & $11.5 \mathrm{~s}$ & $22.8 \mathrm{~s}$ \\
\hline
\end{tabular}

predicted seismograms, while Table 3 lists the values of the time sampling $d t$, the space sampling $d x$, the order $p$ of approximation of the spatial derivatives used and the computational time required. The first seismogram a) is obtained for $d x=3 m$ and $p=4$, the second b) for $d x=27 m$ and $p=4$, the third c) for $d x=27 m$ and $p=p_{l o c}$ and the fourth d) for $d x=27 m$ and $p=24$. Because of the stability condition, we use $d t=0.002 s$ for the seismograms with $d x=27 \mathrm{~m}$ and $d t=0.00025 \mathrm{~s}$ when $d x=3 \mathrm{~m}$.

The first seismogram has a negligible numerical dispersion. On the other hand, a computational time of 57 minutes is needed, because of the large number of non-zero elements in the Laplacian approximation matrix and the very small time sampling imposed by the numerical stability condition. The seismogram in b) needs a computational time of only 6 second, but the approximation error is very high as can be observed comparing the seismograms in a) and in b), due to the numerical dispersion.

The third seismogram in c) is obtained instead through the method we propose, i.e. using a local order of approximation for the spatial derivatives, where the Laplacian matrix is optimized as a function of the velocity model. As described before, the regions of the model with low velocities are modeled by a high order $p_{l o c}$, whereas the regions with high velocities are modeled by a low order $p_{l o c}$. This numerical test requires a computational time of 11.5 seconds, an order of magnitude similar to the second solution test, but results in a low approximation error as we can observed by the similarity between the first and the third seismograms. This last solution represents an optimal trade-off for an inversion procedure, because it shows both small approximation error and low computational time. To validate our results, note that the fourth seismogram in d) computed with a global order of 24 is similar to the third one in c), but the computational time is 


\section{B. Galuzzi, E. Zampieri, E. Stucchi}

22.8 seconds. Finally, assuming the seismogram in a) as the correct solution, we can compute the error of the other seismograms, obtained using $d x=27 m$, and different order $p$ and $p_{l o c}$. In order to consider the approximation error due to the space sampling only, we set $d t=0.00025 \mathrm{~s}$ also for the seismograms corresponding to $d x=27 \mathrm{~m}$, instead of $d t=0.002 \mathrm{~s}$. The error is measured according to the $L^{1}$-norm difference between the seismograms, divided by the $L^{1}$ norm of the seismogram in a).

a)

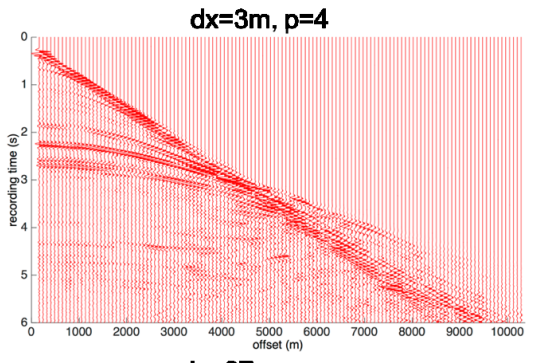

c)

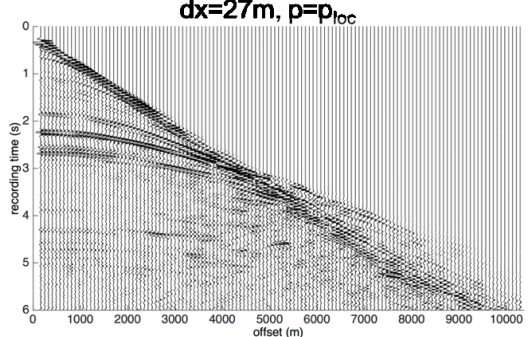

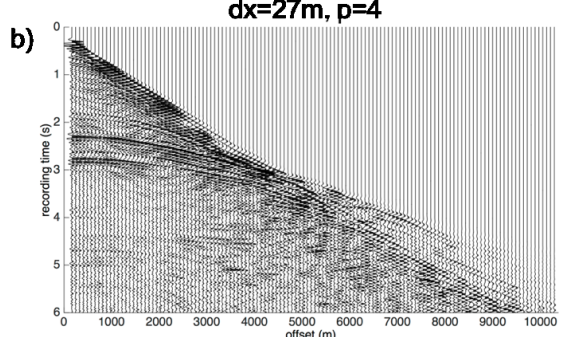

$d x=27 m, p=24$

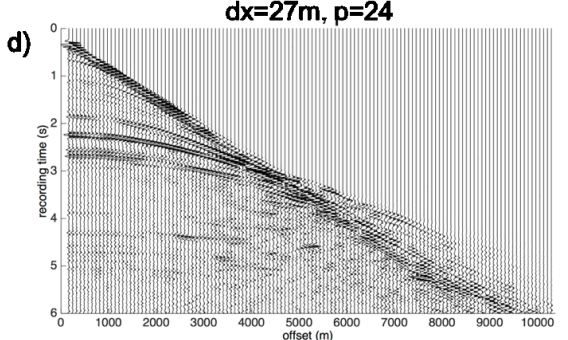

Figure 8. Computed seismograms with different space sampling $d x$ and order of the derivative approximation $p$ : a) $d x=3 m$ and $p=4$; b) $d x=27 m$ and $p=4$; c) $d x=27 m$ and $\left.p=p_{l o c} ; \mathrm{d}\right) d x=27 m$ and $p=24$.

Table 4. relative $L^{1}$-error and computational time of the seismograms with $d x=27 \mathrm{~m}$ and $d t=0.00025 s$, as a function of $p$.

\begin{tabular}{|c|c|c|c|c|c|c|c|c|c|c|c|c|}
\hline$p$ & 4 & 6 & 8 & 10 & 12 & 14 & 16 & 18 & 20 & 22 & 24 & $p_{\text {loc }}$ \\
\hline error & 0.832 & 0.425 & 0.270 & 0.212 & 0.185 & 0.170 & 0.161 & 0.156 & 0.152 & 0.149 & 0.148 & 0.150 \\
\hline time(s) & 37 & 50 & 58 & 68 & 77 & 86 & 95 & 104 & 115 & 124 & 133 & 78 \\
\hline
\end{tabular}

Table 4 lists the values of the errors and the computational time, using a $2.5 \mathrm{GHz}$ Intel Core $i 5$ Processor. If we use higher global orders of $p$, the error decreases, as it can be expected. The $p_{l o c}$-error is comparable to that of higher values of $p$ (between $p=20$ and $p=22$ ), while the $p_{l o c^{-}}$ computational time is between $p=12$ and $p=14$. This example shows that the $p_{l o c}$-method reduces the approximation error and justifies the choice of a local order $p_{l o c}$, rather than of a global one $p$ for the approximation of the spatial derivatives. 


\section{A local adaptive method for numerical approximation in seismic modelling}

\section{Conclusions}

In seismic inversion problems using the numerical solution of the wave equation in order to compute the predicted seismograms, such as in Full Waveform Inversion, reducing the CPU-time necessary for the forward modeling is a key-issue. In fact some inversion procedures, such as the global stochastic optimization routines, require a huge number of forward modelling evaluations to compute the misfit function values and move the solution towards the global minimum. Using a FD implementation for the forward modelling, the accuracy of the solution of the $2 \mathrm{D}$ acoustic wave equation is mainly related to the numerical dispersion inequality. To satisfy this relation, the space sampling $d x$ and the order of approximation of the spatial derivatives $p$, at a given maximum frequency $f_{\max }$ considered in the inversion, are usually set as functions of the minimum acoustic velocity $v_{\min }$. This approach is not efficient, since it requires to use a low $d x$ or a high $p$ even in areas of the model where the velocities are high. The method we propose uses a local order of approximation of the spatial derivatives $p_{l o c}$ rather than a global order $p$, keeping the same forward modelling grid in the different geological areas. This represents an improvement with respect to the classical FD implementation and requires to construct an adaptive stiffness matrix to approximate the Laplacian operator that can be a good compromise between the simplicity of the classical finite difference method and the adaptivity of the finite element method. As a result this method allows to reduce the computational time of the forward modelling, keeping the numerical dispersion low.

As an example of an efficient modelling we simulate a seismic acquisition on the Marmousi velocity model and compute an appropriate solution in terms of efficiency and approximation error, using different combinations of the parameters $d x$ and $p$. Given a fixed grid size $(d x=27 m)$, we conclude that the proposed method can compute a solution in a time that is nearly one half of the time required using $p=24$, and with a similar, low, approximation error.

In conclusion our numerical scheme allows us to model the acoustic wave equation in an efficient way. In particular in an inversion procedure, such as the acoustic Full Waveform Inversion approached with global optimization algorithms, it can be used as an effective tool, tuning the order of approximation of the spatial derivatives $p$ as a function of the velocity model.

The approach studied in this paper represents a first step towards the more complex problem involving large scale $3 D$ domains, where the computational time is even more a critical factor. As a matter of fact, the use of a different $p_{l o c}$ parameter for each grid node does not allow to parallelize 


\section{B. Galuzzi, E. Zampieri, E. Stucchi}

our procedure in an immediate and efficient way. In this respect a suitable reformulation of the algorithm in the framework of parallel implementation could be of interest in the future.

\section{REFERENCES}

1. A. Tarantola, A strategy for nonlinear elastic inversion of seismic reflection data, Geophysics, vol. 51, no. 10, pp. 1893-1903, 1986.

2. J. Virieux and S. Operto, An overview of full-waveform inversion in exploration geophysics, Geophysics, vol. 74, no. 6, pp. WCC1-WCC26, 2009.

3. R. Alford, K. Kelly, and D. M. Boore, Accuracy of finite-difference modeling of the acoustic wave equation, Geophysics, vol. 39, no. 6, pp. 834842, 1974.

4. J. Virieux, V. Cruz-Atienza, R. Brossier, E. Chaljub, O. Coutant, S. Garambois, D. Mercerat, V. Prieux, S. Operto, A. Ribodetti, et al., Modelling seismic wave propagation for geophysical imaging. Intech, 2016.

5. R. E. Sheriff and L. P. Geldart, Exploration seismology. Cambridge university press, 1995.

6. Ö. Yilmaz, Seismic data analysis: Processing, inversion, and interpretation of seismic data. Society of exploration geophysicists, 2001.

7. A. Fichtner, Full seismic waveform modelling and inversion: Springer science \& business media, 2011.

8. A. Sajeva, M. Aleardi, E. Stucchi, N. Bienati, and A. Mazzotti, Estimation of acoustic macro models using a genetic full-waveform inversion: Applications to the marmousi model, Geophysics, 2016.

9. A. Tognarelli, E. Stucchi, N. Bienati, A. Sajeva, M. Aleardi, and A. Mazzotti, Two-grid stochastic full waveform inversion of $2 \mathrm{~d}$ marine seismic data, in 7rth EAGE Conference and Exhibition 2015, 2015.

10. C. L. Liner, Theory of a 2.5-d acoustic wave equation for constant density media, Geophysics, vol. 56, no. 12, pp. 2114-2117, 1991.

11. Z.-M. Song, P. R. Williamson, and R. G. Pratt, Frequency-domain acoustic-wave modeling and inversion of crosshole data: Part iiinversion method, synthetic experiments and real-data results, Geophysics, vol. 60, no. 3, pp. 796-809, 1995.

12. P. R. Williamson and R. G. Pratt, A critical review of acoustic wave modeling procedures in 2.5 dimensions, Geophysics, vol. 60, no. 2, pp. 591-595, 1995.

13. A. R. Mitchell and D. F. Griffiths, The finite difference method in partial differential equations. John Wiley, 1980. 


\section{A local adaptive method for numerical approximation in seismic modelling}

14. K. W. Morton and D. F. Mayers, Numerical solution of partial differential equations: an introduction. Cambridge university press, 2005.

15. R. Richtmyer and K. Morton, Difference methods for initial-value problems. 1967, Interscience, New York.

16. J. C. Strikwerda, Finite difference schemes and partial differential equations. SIAM, 2004.

17. G. C. Cohen, Higher-order numerical methods for transient wave equations, 2003.

18. B. Fornberg, Generation of finite difference formulas on arbitrarily spaced grids, Mathematics of computation, vol. 51, no. 184, pp. 699 $706,1988$.

19. C. Cerjan, D. Kosloff, R. Kosloff, and M. Reshef, A nonreflecting boundary condition for discrete acoustic and elastic wave equations, Geophysics, vol. 50, no. 4, pp. 705-708, 1985.

20. R. Courant, K. Friedrichs, and H. Lewy, On the partial difference equations of mathematical physics, IBM journal of Research and Development, vol. 11, no. 2, pp. 215-234, 1967.

21. C. E. Shannon, Communication in the presence of noise, Proceedings of the IRE, vol. 37, no. 1, pp. 10-21, 1949.

22. C. Bunks, F. M. Saleck, S. Zaleski, and G. Chavent, Multiscale seismic waveform inversion, Geophysics, vol. 60, no. 5, pp. 1457-1473, 1995.

23. A. Brougois, M. Bourget, P. Lailly, M. Poulet, P. Ricarte, and R. Versteeg, Marmousi, model and data, in EAEG Workshop-Practical Aspects of Seismic Data Inversion, 1990. 